\title{
Cosmological Application of Relativity
}

\author{
GEORGES LEMAÎTRE \\ University of Louvain, Belgium
}

\begin{abstract}
The following topics are considered: 1 . Time as coordinate, introduction of proper time through a metric of space time. 2. Geometrical description of matter, the cosmical constant as a second constant demanded by the logical structure of the theory. 3. Detailed computation of the gravitational equations for a general non-static field of spherical symmetry. 4. Schwarzschild's and de Sitter's solutions free from artificial singularities. 5. Map representation of elliptical space, expanding space, Friedmann's equation, reduction to elliptic functions, numerical results. 6. Geodesics of space time, application to proper velocities and redshift. Discussion of the time scale, numerical value of the cosmical constant, data on density. 7. Use of statical coordinates, the instability of Einstein's universe when no restriction is imposed on the symmetry of fluctuations, formation of the nebulae from fast-moving clouds of gases. 8. Discussion of the problem of the clusters of nebulae. 9. The cosmic rays as remnants of primitive matter. Explanation of the large amount of hydrogen and helium in the stars.
\end{abstract}

\section{COORDINATES AND METRIC}

$\mathbf{L}$ ET us consider with Riemann a net of coordinates, i.e., three families of surfaces such that there is one and only one surface of each family which passes through each point of space. If, in each family, the surfaces are specified by the value of some parameter, each point of space is characterized by the values of the three parameters of the three surfaces which pass through it. These three numbers are called the coordinates of the point. These coordinates are defined without any reference to measuring rods.

Einstein extended this conception of Riemann to space time. Let us imagine, that at each point of space, there is a clock, i.e., some mechanism in motion which provides a fourth parameter and enables one to distinguish one from the other, different "events" which occur at that point.

If the clocks are supposed to be identical, i.e., if each of them repeats the same elementary experiment and simply counts the number of the experiments performed, then these clocks show physical time or proper time, time for which it has a meaning to say that the same physical causes take the same time to produce their effects. Proper time plays an essential role in relativity, but it is introduced at a later stage of the theory; the clocks which are involved in the definition of the fourth coordinate of space time need not be physical clocks based on some physical regulating process. They are arbitrarily mechanisms which may be graduated in an arbitrary way. The only assumption which is needed is that the readings of the clocks located in various points of space are continuous functions of the spatial coordinates.

The four coordinates of space time may be varied at will by any continuous transformation. We can replace coordinate time by any other function of the first one and this function may even be different from clock to clock; it may be a function not only of the former time coordinate, but also of the spatial coordinates.
Space coordinates may be varied at will by replacing the original net of three families by any other similar net. Furthermore, the partition of space time into space and time may be altered at will by replacing the threedimensional set of original clocks by another threedimensional set of clocks moving arbitrarily as regards to the first one.

It is important to bear in mind that the coordinates used in relativity have in themselves no compulsory physical meaning, they are just coordinates, time coordinates or spatial coordinates with no physical rigidity; they are what Einstein fittingly called the "mollusc" of reference.

Proper length and proper time are introduced most conveniently from the hypothesis that locally, i.e., in an infinitely small domain of space time, special relativity is valid.

This means that at any point and at any instant, coordinates may be chosen so that, at that point and at that instant, the spatial coordinates are the distances between the corresponding surfaces of the net, and the time is the physical time or proper time of some physical clock based on some regulating mechanism which repeats identical processes.

Furthermore, we must assume that there exists some fundamental velocity (the so-called velocity of light in vacuum) which has the same value for any choice of the motion of the reference clock.

This hypothesis implies that there exists a quadratic form

$$
d s^{2}=-d x^{2}-d y^{2}-d z^{2}+d t^{2}
$$

such that $d s$, counted along the line of space time of the clock, is proper time, and that motion for which $d s=0$ just occurs with the fundamental velocity.

When we return to general coordinates, the quadratic form will be, in general,

$$
d s^{2}=g_{\mu \nu} d x^{\mu} d x^{\nu} \text {. }
$$

The interpretation of $d s$ remains the same, the physical time is $\int d s$ along the trajectory of the clock, and rays of light satisfy $d s=0$. 
Other notions such as simultaneity time and proper length are generalized, in a similar way, from the assumptions of special relativity.

For a family of particles and the corresponding family of lines of space time, lines of simultaneity are defined on this family by the relation

$$
g_{\mu \nu} d x^{\mu} \delta x^{\nu}=0,
$$

where $\delta x^{\nu}$ and $d x^{\mu}$ refer, respectively, to the line of simultaneity and to the trajectories. This is invariant for a change of coordinates and in the case of special relativity where $d x=d y=d z=0$, it gives $\delta t=0$ as it should.

Finally, proper distance of two neighboring particles is the $i d s$ along the line of simultaneity. The length of a line of particles is obtained from the line of simultaneity (defined as a kind of orthogonal trajectory to the space-time trajectories of the points of the spatial line). It is the proper length along this line of simultaneity.

\section{GRAVITATION}

Since special relativity is assumed as an approximation only and not as a rigorous condition, the ten components $g_{\mu \nu}$ of the metrical tensor can be arbitrarily functions of the coordinates.

On the contrary, if they result, by a transformation of coordinates, from a form with constant coefficients, they would satisfy a number of equations; namely, each component of the Riemannian tensor

$$
R_{\nu \sigma}{ }^{\mu \tau}
$$

should vanish. These are well known functions of the $g_{\mu \nu}$ and their derivatives up to the second order, twenty of them are linearly independent, the others being expressed in function of them by simple symmetry conditions.

Besides that, they fulfill four differential equations

$$
\nabla_{\rho} R_{\nu \sigma}{ }^{\mu \tau}+\nabla_{\nu} R_{\sigma \rho}{ }^{\mu \tau}+\nabla_{\sigma} R_{\rho \nu}{ }^{\mu \tau}=0,
$$

where $\nabla$ means the covariant derivative. When coordinates are changed arbitrarily, these quantities transform according to rules indicated by the position of the indices, rules which are codified in the tensor calculus. A tensor is nothing else than a set of quantities which transform according to well defined rules. When a tensor vanishes for some coordinates it vanishes for all coordinates. The corresponding set of equations is said to be invariant for a change of coordinates.

From the Riemannian tensor it is possible to deduce other tensors, the so-called contracted tensor

$$
R_{\nu}{ }^{\mu}=R_{\nu \sigma}{ }^{\mu \sigma}
$$

and the totally contracted tensor

$$
R=R_{\nu}{ }^{p} \text {. }
$$

From the differential equation satisfied by the Riemannian tensor, four corresponding identities can be deduced for the contracted tensor

$$
\nabla_{\nu}\left(R_{\mu}{ }^{\nu}-\frac{1}{2} g_{\mu}{ }^{\nu} R\right)=0 .
$$

The idea of Einstein's theory of gravitation is that matter is fully described by the metrical tensor and the corresponding Riemannian tensors.

In order to explain this connection, i.e., the equation of gravitation, we first notice that matter cannot be represented by the full Riemannian tensor; otherwise there would be no possibility for a gravitation field to exist in a place where no matter is present; components of the Riemannian tensor may be different from zero without involving the presence of matter. This leads us to identify matter with the contracted Riemannian tensor and not with the whole tensor.

But actual matter exhibits some permanency; this leads us to recognize as matter the combination which occurs in the identity satisfied by the contracted Riemannian tensor only. In that way, even if we suppose that the $g_{i k \nu}$ vary in any way, the tensor which we recognize as representing matter shall behave in a plausible way which will imitate conservation and causality.

This involves that the tensor which represents matter satisfies the relation

$$
-R_{\mu}{ }^{\nu}+\frac{1}{2} g_{\mu}{ }^{\nu} R=\kappa T_{\mu}{ }^{\nu}+\lambda g_{\mu}{ }^{\nu},
$$

Einstein's gravitational equations, involving two constants. The $\lambda$-term may be introduced because it does not disturb the identity.

Then the material tensor satisfies the four identities

$$
\nabla_{\nu} T_{\mu}{ }^{\nu}=0 .
$$

Coordinates may be chosen so that, at some prescribed point, these equations reduce to

$$
\partial T_{\mu}{ }^{4} / \partial t+\partial T_{\mu}{ }^{1} / \partial x+\partial T_{\mu}{ }^{2} / \partial y+\partial T_{\mu}{ }^{3} / \partial z=0 .
$$

This would be exact only at the prescribed point and would involve, in other places, an error which is small in the immediate neighborhood of this point.

Using Green's theorem, we find this means that the time derivative of

$$
\int T_{\mu}^{4} d V
$$

extended to some volume $V$, is equal to the outgoing flux of the vector of components $T_{\mu}{ }^{1}, T_{\mu}{ }^{2}, T_{\mu}{ }^{3}$ through the boundary of the volume $V$.

This is the way in which conservation is expressed mathematically. For $\mu=4$ this means conservation of a scalar quantity, which must be identified with the density of matter, while the three other values of $\mu$ are the three components of a vectorial quantity, the density of momentum. 
Elementary tensor relations show that this density of momentum is precisely the flux of the density.

For both indices different from 4, we get a representation of the stresses in matter used in the theory of elasticity. In fact, it is from this particular application that the word tensor has been formed.

When the $d s^{2}$ contains square terms only, i.e., when

$$
d s^{2}=\sum_{k}\left(a_{k} d x^{k}\right)^{2}
$$

where the $a_{k}$ are functions of the four coordinates $x^{i}$, the computation of the Riemannian tensor can be made very easily. ${ }^{1}$

The components with four different indices vanish. Those with three different indices are $\left(\partial_{k}\right.$ means $\left.\partial / \partial x^{k}\right)$

$$
R_{i i k l}=a_{i}\left(\partial_{k l}{ }^{2} a_{i}-\partial_{k} a_{i} \cdot \partial_{l} a_{k} / a_{k}-\partial_{l} a_{i} \cdot \partial_{k} a_{l} / a_{l}\right),
$$

and those with two different indices can be conveniently written

$$
R_{i k}^{i k}=\beta_{i k}=\beta_{k i}
$$

where

$$
\begin{aligned}
\beta_{i k}=\left[\partial_{i}\left(\partial_{i} a_{k} / a_{i}\right)+\partial_{k}\left(\partial_{k} a_{i} / a_{k}\right)\right. & \\
& \left.+\sum_{l} \partial_{l} a_{i} \cdot \partial_{l} a_{k} / a_{l}{ }^{2}\right] / a_{i} a_{k} .
\end{aligned}
$$

This expression is homogeneous in the indices. This comes from the fact that the $d s^{2}$ admits of a transformation in which the $a_{k}$ are each multiplied by some constant and the corresponding $x^{k}$ divided by the same constant.

It is therefore always possible to choose the $a_{k}$ equal to one, at the point where we intend to make the computation. Practically, as soon as all derivations have been performed, we can simply omit the $a_{k}$. This circumstance can be used to shorten the computation. The $a_{k}$ can be restored in the final result from the condition of homogeneity.

The case where the components with three different indices vanish is of special interest. In that case, the identity satisfied by the Riemannian tensor takes the simple form

$$
\partial_{l} \beta_{i k}+\left(\beta_{\imath k}-\beta_{l k}\right) \partial_{l} a_{i} / a_{i}+\left(\beta_{i k}-\beta_{l 2}\right) \partial_{l} a_{k} / a_{k} .
$$

The corresponding values of the contracted tensor are

$$
R_{i}^{i}=\sum_{k} \beta_{i k},
$$

and the gravitational equation gives for equal indices

$$
\lambda+\kappa T_{n}^{n}=\beta_{i k}+\beta_{i l}+\beta_{k l} .
$$

When the three-indices-components of the Riemannian tensor vanish, as we have supposed, then the other components $T_{i}^{k}$ vanish.

\footnotetext{
${ }^{1}$ Details of these computations may be found in the mathematical appendix of Lemaître, L'hypothese de l'atome primitif. Le Griffon (Neufchatel, 1946). For Latin indices we do not use Einstein's summation. Different letters mean different values of the indices.
}

\section{SPHERICAL SYMMETRY}

Let us consider a bundle of lines and define angular coordinates in the customary way, for instance, $\theta$ as a zenith distance, $\varphi$ as an azimuth.

Then the angle $d \sum$ of two neighboring rays is given by

$$
d \sum^{2}=d \theta^{2}+\sin ^{2} \theta d \varphi^{2} .
$$

This is a formula of Euclidean geometry, we can assume it because Euclidean geometry is valid in the infinitesimal neighborhood of any point.

The bundle of lines admits of a group of transformation turning $\theta$ and $\varphi$ into new coordinates $\theta^{\prime}$ and $\varphi^{\prime}$ for which the expression $d \sum$ is precisely the same as before, but with $\theta$ and $\varphi$ replaced by $\theta^{\prime}$ and $\varphi^{\prime}$. This group of transformation is simply the Euclidean group of rotations round the center of the bundle. If the $d s$ can be written in such a way that it remains invariant for this group of transformation we say that there is spherical symmetry.

This occurs if

$$
d s^{2}=-a^{2} d \chi^{2}-r^{2} d \sum^{2}+c^{2} d t^{2},
$$

where $a, r$, and $c$ are function of $\chi$ and $t$ only.

If we make any change of coordinates turning $\chi$ and $t$ into $\chi^{\prime}$ and $t^{\prime}$, spherical symmetry will be preserved. Terms in $d \chi d t$ need not be considered because such terms can always be suppressed by a convenient choice of the transformation. This involves a restriction on the choice of the coordinates but not a restriction on the generality of the field represented.

This restriction does not exhaust the freedom we have to choose another coordinate system. If $\chi^{\prime}$ is a function of $\chi$ and $t$, then $t^{\prime}$ is determined as a function of $\chi$ and $t$ in such a way that the lines $t^{\prime}=$ constant are simultaneity lines on the family of lines for which $\chi^{\prime}$ is a constant.

We may utilize the remaining freedom of coordinates in order that the components with three different indices of the Riemannian tensor would vanish.

In fact, for spherical symmetry, each of these components vanishes identically with the exception of

$$
R_{214^{2}}=R_{314^{3}} \text {, }
$$

and these will vanish for a convenient choice of the coordinates.

Then the component $T_{1}{ }^{4}$ of the material tensor vanishes. This means that there is no momentum along the radii; the motion of the reference "mollusc" is so adjusted that it follows exactly the mean motion of the matter, so that there is no net momentum across the moving sphere $\chi=$ constant.

There may still be some to and fro transfer of momentum; this is exhibited in the component $T_{1}{ }^{1}$.

Using the formula (2.14) found for this case, we have

$$
\partial_{l} \beta_{23}+\left(2 \beta_{23}-\beta_{2 l}-\beta_{3 l}\right) \partial_{l} r / r=0,
$$

and the corresponding gravitation equation is (with 
$l, n=1,4$ or 4,1$)$

$$
\kappa r^{2} T_{n}^{n} \partial_{l} r=\partial_{l}\left(r^{3} \beta_{23}-\lambda r^{3} / 3\right) .
$$

Let us write

$$
T_{4}{ }^{4}=\rho, \quad T_{1}{ }^{1}=-p, \quad T_{2}{ }^{2}=T_{3}{ }^{3}=-\tau,
$$

so that $\rho$ is the density, $p$ is the radial pressure, $\tau$ the transversal pressure, and introduce a function $m$ of $\chi$ and $t$ defined by

$$
\kappa m / 4 \pi=r^{3} \beta_{23}-\lambda r^{3} / 3 \text {. }
$$

Then, $\beta_{23}$ is found to be

$$
r^{2} \beta_{23}=1-(\partial r / a \partial \chi)^{2}+(\partial r / c \partial t)^{2}
$$

so that the definition of $m$ amounts to

$$
(\partial r / c \partial t)^{2}=-1+(\partial r / a \partial \chi)^{2}+\kappa m / 4 \pi r+\lambda r^{2} / 3,
$$

which is the energy equation for the motion in $r$.

We have also

$$
\begin{aligned}
& 4 \pi \rho r^{2} \partial r / \partial \chi=\partial m / \partial \chi \\
& 4 \pi p r^{2} \partial r / \partial t=-\partial m / \partial t .
\end{aligned}
$$

The condition introduced for the choice of the coordinates $\left[R_{1422}=0(2.11)\right]$ is

$$
\partial(\partial r / a \partial \chi) / \partial t=\partial r / c \partial t \cdot \partial c / a \partial \chi .
$$

Finally, we write the conservation identities

$$
\begin{array}{r}
\partial p / \partial \chi+2(p-\tau) \partial r / r \partial \chi+(\rho+p) \partial c / c \partial \chi=0 \\
\partial \rho / \partial t+2(\rho+\tau) \partial r / r \partial t+(\rho+p) \partial a / a \partial t=0 .
\end{array}
$$

These are the equations for the general field with spherical symmetry. ${ }^{2}$

\section{SCHWARZSCHILD AND DE SITTER SOLUTIONS}

The case where $p$ and $\tau$ vanish is of special interest. In that case there is no interior stress, and matter is formed of individual particles which move without crossing each other. Surfaces can be defined which move in such a way that each material particle remains in the same cell of the net.

In that case $m$ is a function of $\chi$ only, and $c$ of $t$ only. Then $c$ can therefore be assumed to be unity (see (5.4)).

Furthermore, $\partial \zeta / a \partial \chi$ is a function of $\chi$ only, say $f(x)$, and the field is defined by

$$
d s^{2}=-(\partial r / \partial \chi)^{2} d \chi^{2} / f^{2}(\chi)-r^{2} d \sum^{2}+d t^{2},
$$

the function $r$ of $\chi$ and $t$ satisfies Eq. (3.9),

$$
(\partial r / \partial t)^{2}=-1+f^{2}(\chi)+\kappa m(\chi) / 4 \pi r+\lambda r^{2} / 3 .
$$

The solution of this equation depends on elliptic functions and will be considered later on in connection with Friedmann's equation. In the case $f(\chi)=I$, the solution

\footnotetext{
2 These formulas have been obtained in a somewhat different way in G. Lemaître, L'univers en expansion, Annales de la société scientifique de Bruxelles, (1933), Tome 53, pp. 51-85, reproduced in "Publications du laboratoire (d'Astronomie et de Géodésie de l'Université de Louvain” IX (1932).
}

is elementary, it is

$$
r=r_{0} \sinh ^{\frac{2}{3}} \alpha\left(t-t_{0}\right),
$$

where $\alpha$ is a constant

$$
\alpha^{2}=9 \lambda / 4 .
$$

$r_{0}$ is the function of $\chi$ :

$$
r_{0}^{3}=3 \kappa m(\chi) / 4 \pi \lambda \text {. }
$$

$t_{0}$ is an arbitrary function of $\chi$.

The origin is a singular point from which material particles are continuously ejected, it is a source of particles and could be called a source of space, as new space is introduced with the new particles.

The geometry of observers operating on these particles is obtained by putting $d t=0$ because $t$ is obviously simultaneity time for the set of particles, the spatial element $d \sigma$ is therefore given by

$$
d \sigma^{2}=(\partial r / \partial \chi)^{2} d \chi^{2}+r^{2} d \chi^{2}
$$

or for $d t=0$, by

$$
d \sigma^{2}=d r^{2}+r^{2} d \sum^{2}
$$

This is the Euclidean line element written with polar coordinates. Therefore, geometry is Euclidean on the expanding set of particles which are ejected from the point singularity at the origin.

If we suppose that $\rho$ is infinitely small, we get, as a limiting case, the field of a point singularity at the origin. In that case, the particles continuously ejected have no physical reality; they are just mathematical devices introduced in order to define the coordinates and the corresponding partition of space and time.

$m$ reduces to a constant, and therefore, $r_{0}$ is also a constant. The particles with different $\chi$ must start at different epochs, otherwise their motion would be identical. It is therefore possible to choose the date of birth of the particle as the coordinate $\chi$.

Writing $t_{0}=\chi$, we have, from (4.3) and (4.4),

$$
d r^{2}=\lambda\left(r^{3}+r_{0}^{3}\right)(d t-d \chi)^{2} / 3 r
$$

and substituting $d \chi$ in $d s^{2}$ (4.1),

$$
\begin{aligned}
d s^{2}=-d r^{2}-r^{2} d \sum^{2}+2 \lambda\left(r^{3}+r_{0}^{3}\right) d \chi d t / 3 r \\
+ \\
+\left[1+\lambda\left(r^{3}+r_{0}^{3}\right) / 3 r\right] d t^{2} .
\end{aligned}
$$

Let us introduce a new coordinate $\tau$ by $^{3}$

$$
\tau=t+\lambda^{\frac{1}{2}} \int\left[\left(r^{3}+r_{0}^{3}\right) / 3 r\right]^{\frac{1}{2}} d r /\left[1-\lambda\left(r^{3}+r_{0}^{3} / 3 r\right] .\right.
$$

Substituting $d t$ in $d s^{2}$ we get

$$
\begin{aligned}
d s^{2}=-d r^{2} /\left[1-\lambda r^{2} / 3-\lambda r_{0}^{3} / 3 r\right]-r^{2} d \sum^{2} & \\
+ & {\left[1-\lambda r^{2} / 3-\lambda r_{0}{ }^{3} / 3 r\right] d \tau^{2} . }
\end{aligned}
$$

This is the well known Schwarzschild solution. It shows that Einstein's constant $\kappa$ must be identified

${ }^{3}$ When the cosmical constant vanishes, $\tau$ may be found in finite form (reference 2, formula 11.10). 
with Newton's constant of gravitation by the wellknown formula, $\kappa=8 \pi G / c^{2}$.

If we introduce natural coordinates, i.e., $c=I$ and $G=I$, we can write

$$
\kappa=8 \pi \text {. }
$$

If $m$ tends to zero, then $r_{0}$ also tends to zero and we have

$$
r=r_{1} e^{2 \alpha t / 3}
$$

where

$$
2 r_{1}=\lim r_{0} e^{-2 \alpha t_{0} / 3} \text {. }
$$

$t_{0}$, date of birth of the particles, is removed to minus infinity.

No modification occurs in the analysis and we get from (4.1) and (4.13)

$$
d s^{2}=-e^{4 \alpha t / 3}\left(d r_{1}^{2}+r_{1}^{2} d \sum^{2}\right)+d t^{2}
$$

and in statical coordinates as in (4.11)

$$
d s^{2}=-d r^{2}\left(1-\lambda r^{2} / 3\right)^{-1}-r^{2} d \sum^{2}+\left(1-\lambda r^{2} / 3\right) d \tau^{2}
$$

which is the original form of the de Sitter universe, with its well-known singularity at the horizon of the center. It may be seen that de Sitter's singularity like Schwarzschild's singularity is an artificial singularity, not of the field but of the coordinates introduced to describe this field.

\section{EXPANDING SPACE-FRIEDMANN'S EQUATION}

Let us consider a bundle of lines, and in this bundle a cone, i.e., a closed family of lines starting from a line and coming back to it.

If it comes back to the original line in the direction opposite to the original one, we call the cone an odd cone. If it comes back with the original direction, it is an even cone. The reason for these names is that if an odd cone is supposed to be described twice before closing it becomes an even cone.

An even cone can be deformed continuously so that it shrinks into a single line. Odd cones cannot. If they are stretched as strongly as they can be, they become flattened into planes.

Bundles of lines can be studied on a small sphere whose center is the center of the bundle. This sphere is supposed to be small in order that it might be assumed to have Euclidean properties.

Each line of the bundle is represented by one of the two antipodical intersections with the sphere. Angles of two lines are measured by the length of the corresponding arcs, angles of planes of the bundle by angles of circles on the sphere.

In order to get a true representation, so that each line of the bundle would correspond to one and only one point on the map, we must restrict the representation to a portion of the sphere. This portion may be drawn in many ways; the simplest one being to use an hemisphere.
On the limiting great circle which is the boundary of the representation, lines of the bundle will be represented twice. When we reach the boundary, and would have to go out of the map, we use the other representing point, and enter the map from this opposite point.

If we allow continuous deformation of the hemisphere, it can be flattened down on a small Euclidean disk. The line of angular coordinates $\theta$ and $\varphi$ will be represented by a point at a distance $\theta$ in the direction $\varphi$, and the formula

$$
d \sum^{2}=d \theta^{2}+\sin ^{2} \theta d \varphi^{2}
$$

will define the scale of the representation at each point and in every direction, $d \sum$ being the angle between neighboring lines.

The map is closed by the circle $\theta=\pi / 2$ and coordinates $\theta=\pi / 2 . \varphi=\varphi_{0}$ or $\theta=\pi / 2, \varphi=\varphi_{0}+\pi$ represent the same line of the bundle.

This geometrical construction can be extended from two to three dimensions. The map is then defined as follows. Consider a bundle of rays with angular coordinates $\theta$ and $\varphi$ (we say rays and not lines because opposite half-lines must now be distinct) and on each of these rays, points at a distance $\chi$ from the center of the representation. Scales of the representation are defined in such a way that the true angular distance $d \sigma$ is given by

$$
d \sigma^{2}=d \chi^{2}+\sin ^{2} \chi d \sum^{2},
$$

$d \sum$ being the same expression as before.

Mathematically all we have said for the two-dimensional case can be repeated without any essential change. Of course, the intuitional background is lacking. We have no intuition of a space with four dimensions. Nevertheless, we may speak of representing a hyperbundle of lines in a four-dimensional Euclidean space.

This would not clarify our notions as easily as before, however, it provides a proof of the homogeneity of the geometry obtained. It shows that the point represented at the center has no special property and that displacements can be defined which would turn any point into the center of the representation, leaving the representation exactly the same as for the first center.

We have thus obtained a geometry where there is spherical symmetry around any point of space, a geometry in which every point and every direction is equivalent to any other, a space homogeneous and isotropic.

Its definition and its representation has been given by particularizing a general Riemannian geometry. This geometry is also called Riemann geometry or elliptical geometry.

The whole space is represented inside the sphere of radius $\pi / 2$. Opposite points on the boundary represent a unique real point. A line is represented by opposite rays which are sealed into one closed segment, by the point represented twice on the boundary. 
The total length of any straight line is therefore equal to $\pi$.

These notions may be applied to space time. In order that spherical symmetry should exist around any point, we must particularize the results found for spherical symmetry, so that the three spatial coordinates $\theta, \varphi, \chi$ would appear in the expression $d \sigma$ only. This means that we must put

$$
a=R \quad r=R \sin \chi,
$$

in the formula of spherical symmetry. $R$ is a function of the fourth coordinate $t$ only.

$c$ could be a function of $t$, but we do not loose any generality by choosing it equal to one. This can be achieved by a transformation

$$
t^{\prime}=\int c d t
$$

we obtain Friedmann's expression

$$
d s^{2}=-R^{2} d \sigma^{2}+d t^{2} .
$$

It involves a partition of space and time and a set of clocks at each node of the net of the spatial coordinates, and we may speak of conceptual observers which read the clocks and handle measuring rods.

From the group of displacements involved in the geometry, it follows that observations of these observers would be exactly similar, they have precisely the same general outlook of the universe around them.

The coordinates used are such that $t$ is not only coordinate time but also simultaneity time and proper time because $d s=d t$ for $d \sigma=0$. This would not be the case if we had replaced $t$ by some functions of $t$ so that $c$ would not be a constant. Therefore, $t$ is a physical time and if something really happens, if some atom emits or absorbs light, the frequency or wave-length of the light will have its standard value, when reckoned in time $t$. As explained before, this is the meaning of proper time.

The proper length is $R \sigma$. It is the product of two factors, an angular distance $\sigma$ which remains constant for the standard observers whose distribution into the whole space remains the same. This constant angular distance is multiplied by a variable factor $R$ in order to be converted into linear measure. $R$ is called the radius of space, and we have obtained a space of variable radius. The tour of the line is $\pi R$. When it increases with time, we get an expanding space.

'For these special values of $c$ and $r$ we have

$$
\partial r / a \partial \chi=\cos \chi .
$$

$m$ must be such that

$$
m=M \sin ^{3} \chi .
$$

Where $M$ is a function of $t$ only which may be deter- mined by Friedmann's equation,

$$
(d R / d t)^{2}=-1+2 M / R+R^{2} / T^{2}
$$

obtained from (3.9). We have written

$$
\lambda / 3=1 / T^{2} .
$$

The density $\rho$ is given by

$$
M=4 \pi \rho R^{3} / 3 .
$$

$p$ and $\tau$ can be computed from

or

$$
4 \pi p R^{2} d R / d t=-d M / d t
$$

$$
d \rho / d t+3(\rho+p) d R / R d t=0 .
$$

In most astronomical applications $p$ is small in comparison with $\rho$ and $M$ is approximatively a constant.

In order to study the Friedmann equation, it is convenient to use the angular distance $\sigma$ which has been traveled by light during the time $t . \sigma$ may be used as a time coordinate in place of $t$.

In terms of $\sigma$, Friedmann's equation becomes

$$
(d R / d \sigma)^{2}=-R^{2}+2 M R+R^{4} / T^{2} .
$$

Its solution is

$$
\text { with } \begin{aligned}
1 / R & =1 / 6 M+p(u) \\
\sigma^{2} & =2 \cdot u^{2} / M,
\end{aligned}
$$

where the elliptic function has invariants

Writing

$$
g_{2}=1 / 3 M^{2}, \quad g_{3}=1 / 27 M^{3}-2 / M T^{2} .
$$

we have

$$
\mathfrak{p}(v)=-1 / 6 M,
$$

$$
t / T=-2 u \zeta(v)+\log \sigma(u+v) / \sigma(v-u) .
$$

Standard methods of computation of elliptic function may be used. Details may be found in reference 2, Eq. (9.33) where approximate formulas sufficient for most astronomical applications are given (10.14).

It must be possible also to use the Bartky method of computation which has been extended to non-complete integrals. ${ }^{4}$

\section{GEODESICS - PROPER VELOCITIES - RED SHIFT}

A material particle, free to travel through the universe, would describe a geodesic of space time. This would be a straight line of angular space, traveled with a velocity which is obtained by the condition that $\delta \int d s$ is an extremum.

This means that $R^{2} d \sigma / d s$ remains constant.

Let us introduce in this expression the linear velocity $v=R d \sigma / d t$ and the mass $m=m_{0} d t / d s$ where $m_{0}$ is the proper mass; we obtain directly, $m v=C / R$.

${ }^{4} \mathrm{G}$. Lemaître, Caleul des intégrales elliptiques, Bulletin de l'Académie royale de Belgique (classe des sciences) 34, 200 (1947). W. Bartky, Rev. Mod Phys. 10, 264 (1938). 
This means that the momentum of the particle varies as $I / R$.

This formula can be applied to light, although this case is easily obtained directly. This shows that light which has been emitted at a time $t_{1}$ with a frequency $\nu_{1}$, when the radius of space was $R_{1}$, it is received at a time $t_{2}$ of radius $R_{2}$ with a frequency $\nu_{2}$ such that $\nu_{1} R_{1}$ $=\nu_{2} R_{2}$. If this light is compared with a source exactly similar but operating now with the frequency $\nu_{1}$ (the same because it is reckoned in proper time), then this light is observed as reddened in the ratio $R_{2} / R_{1}$ of the expansion which did occur during its transmission.

For moderate red-shifts which are given by the astronomers as spectroscopic velocities $v$ the value $v / c=d \lambda / \lambda=\left(R_{2}-R_{1}\right) / R_{1}$ is not very different from $t d R / R d t$, then the red-shift is proportional to the linear distance $c t$ of the source. The ratio $c t / v=T_{H}$ has been observed; its value is $2 \times 10^{9}$ years. (HubbleHumason.)

These results may be interpreted geometrically. If we consider a graph of the values of $R$ plotted against the time, the subtangent of the curve at the point corresponding to the actual observations must have the value $T_{H}$.

Whatever may be the solution of Friedmann's equation we adopt, this value of the subtangent $T_{H}$ gives the scale of time. Its amount is probably shorter than the duration of geological ages. We cannot hope to get a time available for the evolution much greater than $T_{H}$ but we must have it somewhat greater.

This means that the curve $t, R$ must have its concavity turned upwards or that the second derivative of $R$ must be positive. Its value is

$$
d^{2} R / d t^{2}=-M / R^{2}+\lambda R / 3 .
$$

This is the first instance we connect the cosmological constant with observation. At the start, it was a constant demanded by the logical deduction of the gravitational equation. All logical requirements could be satisfied by an equation containing two available constants only. One of these constants $\kappa$ was identified with Newton constant of gravitation. For the other $\lambda$, all we knew from the observation of planetary motions was that its value must be very small. It is only in phenomena involving large distances that the small term containing the cosmological constant has significant effects, and it is on this account that it has received its name.

The cosmological constant must be positive and its value must be sufficient to overcome the negative term in $M$ in (6.1). This justifies the notation we have used in anticipation, (5.9).

Comparing Friedmann's equation with the expression of the red-shift, we see that the $T$ involved in the cosmological constant cannot be very different from the $T_{H}$ given by the observations.

The condition that the acceleration is positive can be transformed into a relation between the cosmo- logical constant and the density. Using the relation between $M$ and $\rho$, we find this condition is that the density should be greater than a value $\rho_{0}$ which might be called the cosmological density, and which is related to the cosmological constant by the formula

$$
4 \pi \rho_{0}=3 / T^{2}=\lambda .
$$

For $T=T_{H}$ the value of $\rho_{0}$ is $\rho_{0}=10^{-27}$ in c.g.s. units.

The mean value of the density may be deduced from the observation of the rotation of the biggest extragalactic nebulae and the average distance of these nebulae, it is $\rho=10^{-30}$. This justifies the interpretation of $T_{H}$ as an estimate of $T$ and shows that the value $R_{E}$ of $R$ for which the acceleration did vanish was about ten times smaller than its value today because

$$
R / R_{E}=\left(\rho / \rho_{0}\right)^{\frac{1}{3}} .
$$

If the ratio $M / T$ has precisely the value $3^{-\frac{2}{3}}$ for which the quartic of Friedmann's Eq. (5.13) has a double root, then the radius $R$ would take an infinite time to vary from $R_{E}$ to $R$.

If this condition is approximated, let us say to within one in a thousand, then this same duration from $R_{E}$ to $R$, reckoned in units of $10^{9}$ years, is equal to 9 .

If the approximation is within one in a million, the corresponding duration is 13 , in the same units. In the case of imaginary roots, the total time from the zero value of the radius is 13 in the first case, and 17 in the second case. This last figure may therefore be considered as an extreme limit to any cosmology using this solution of Friedmann's equation.

\section{INSTABILITY}

The use of an angular space is very convenient when we wish to emphasize the general homogeneity of spacetime. But the perfect flexibility of relativity allows of the use of any kind of coordinates and for some problems, it is very convenient to use statical coordinates.

Of course we must not forget that they are coordinates only and that their metrical properties must be inferred from inspection of the $d s$. If we think of them in terms of metric and time, it is only in the sense of a map-representation, involving scale correction which may be very large and also boundary conditions such as we have obtained for elliptic geometry.

In this type of representation the standard particles, uniformly distributed in the angular space, are represented at a variable distance $r=R \chi$ from the origin. We call their velocity in this representation, the standard velocity. This is an altogether different velocity from the proper velocity, which we have considered when we spoke of the free particles moving along geodesics of space time. In static representation, such proper velocity should be represented as an addition to the standard velocity.

We have seen that the proper velocity diminishes as $1 / R$. This may be interpreted in the statical repre- 
sentation. Particles which have velocities different from the standard one at the place where they are, will progressively reach places where the standard velocity is not so different from their own velocity, the difference which is their proper velocity is accordingly reduced.

Particles with angular distance $\pi / 2$ from the origin are represented as an expanding sphere of radius $\pi R / 2$, which is the boundary of the representation and the locus where the opposite rays are sealed into a closed line by a point which is represented twice at antipodal points of the map.

These points which, on the representation, appear to be at the end of the world are of course simply on the edge of the map.

When we consider a region which is not too large as regards the radius of space, say much smaller than $10^{10}$ light years, then the scale deformations are not conspicuous and this representation is very useful.

It is useful also because it allows us to get rid of conditions which have been introduced for mathematical convenience but which are not strictly realized in nature. I mean the condition of symmetry, spherical symmetry, or homogeneity; these are probably large scale features of the universe, but they cannot be assumed to be realized exactly.

If we consider a region small enough, the standard velocities will not be great compared to the velocity of light, so when the variation of mass with velocity and all phenomena of this kind can safely be ignored, it is then possible to use a mechanical description of the evolution of these particles, even if this representation departs locally very strongly from any kind of symmetry.

It is well known that in this approximation Newtonian mechanics is a good approximation to relativity. All we need is to know, in the Newtonian approximation, the effect of the cosmological constant.

This is extremely simple. The Newtonian approximation has been obtained by Einstein, under the approximation that the only significant part of the material tensor, for the coordinates involved in the representation, is $T_{4}^{4}=\rho$. The effect of the cosmological constant is simply to replace $\rho$ by $\rho-\rho_{0}$ where $\rho_{0}$ is the cosmological density introduced above.

In working out Newtonian mechanics, we need only to replace $\rho$ by $\rho-\rho_{0}$ in Poisson's equation.

When the radius of space has the equilibrium value $R_{E}$, then, in the mean, the effect of gravitation is canceled. But if there are local fluctuations of the density around its mean value $\rho_{0}$, the field will divide itself, as a result of these fluctuations, into repellent and attractive regions.

The significance of the instability of the equilibrium is not only that small departures from equilibrium will decide whether the radius will increase or decrease. This is the only effect which is allowed by the symmetry condition. But when this condition is removed, contraction may prevail in some places and dilatation in other places, so that, if the mean expansion prevails, it may be that contraction occurs locally leaving finally contracting regions which depart one from the other in an expanding space.

If, therefore, the condition for double roots fails to be satisfied, so that the roots are slightly imaginary, then when the radius of space was slowly surpassing the equilibrium radius, these phenomena of instability have been very important. They can be studied locally in the statical representation by methods of ordinary mechanics with due regard to the modification of Poisson's equation.

Besides the modification of Poisson's equation, another feature of these applications of classical mechanics must not be forgotten.

It is very natural to use, in connection with astronomical systems, notions taken from the theory of gases.

We deal with super gases whose "molecules" are stars or nebulae, diffuse nebulae, i.e., clouds of ordinary gases or even enormous systems such as the extragalactic nebulae. This is, of course, what we have done when we spoke of density and pressure in the material tensor. In these applications, pressure is simply a way of accounting for the mean velocity of the molecules.

The analogy with gases might be pressed a little further, in the sense that effects of averaging by mixture might occur and be pictured by what is called dynamical equilibrium. But there are essential differences. The regulating effect which, in a gas is due to the collisions, has no counterpart in the astronomical problem. In other words, we are dealing with cases of extremely rarefied gases in which effects of collisions must be ignored. Even this could only be accepted with caution as collisions with the walls of the vessel would still establish some kind of a Maxwellian distribution of velocities, and this would have no counterpart in the astronomical problem.

In our case, molecules may have individual effects. If some large molecule exists in the unstable equilibrium, its gravitational attraction will retain all other molecules which pass in its neighborhood with velocity not too different from its own. The resulting condensation will have the velocity of the original abnormally large molecule.

Great proper velocities of the resulting condensations are a manifestation of the large velocities of the original "molecules" round which they aggregate.

The final distributions of the velocities in the system formed will result in an averaging in phase space. The density in phase space will be approximatively uniform, but the total volume occupied will be restricted to velocities inferior to the velocity of escape.

This distribution has been studied in the case of spherical symmetry. ${ }^{5}$ The density distribution is very

${ }^{5}$ G. Lemaître, "Un modèle de nébuleuses," Ann. de Soc. Sci. de Bruxelles 60, 103 (1946). 
different from the distribution in elliptical nebulae. There is no marked condensation in the central region. Also the dimensions would be much greater. The central density is only seven times the cosmological density.

It is therefore clear that, while such a distribution has been an intermediary stage in the evolution of a nebula, modifications did occur later, involving a loss of energy so that a strong central condensation might result from it.

This process will come out from non-elastic collisions between the original molecules. Molecules colliding with opposite velocities will be reduced to rest and fall together towards the center.

It seemed of interest to work out, as an extreme case, a model where the motion of the molecules is exclusively radial. This has been done with two hypotheses of the distribution of the velocity. ${ }^{6}$

It turns out that the distribution has a singularity at the center which imitates the nucleus observed in nebulae. The distribution of density is very similar to the observed one, this observed distribution being an intermediary between the two models which have been studied.

A consequence of this requirement of non-elastic collisions between the original molecules is that these molecules cannot be stars. It is well known that in astronomical conditions collisions of stars are utterly negligible within a duration of a few $10^{9}$ years. We must therefore picture these molecules as something more or less similar to diffuse nebulae; they are clouds of ordinary gas or dust. If such clouds collide with velocities of the order of a few hundred kilometers a second, the heat produced will be dissipated by radiation. Of course stars would be expected to result, as an agglomerate of these original clouds.

\section{CLUSTERS OF NEBULAE}

The extra-galactic nebulae are distributed in a rather irregular way showing a large clustering tendency and occasionally great clusters of a few hundred nebulae congested within a sphere of less than one million light years of radius.

In these clusters the nebulae are at distances ten times smaller than those of field nebulae. The density is not very different from the cosmological density $\rho_{0}$.

Clusters may be considered as regions which are still in (or near) the unstable equilibrium so that, in the mean, there is no net force coming out of the contest between the cosmical repulsion and Newtonian gravitation.

We must therefore accept the suggestion put forward by de Sitter in $1933 .^{7}$ It would appear that existing

${ }^{6}$ G. Lemaître and R. Vander Borght, "Modèles de nebuleuses à vitesses radiales," Bull. de Acad. Roy. de Belgique, classe des sciences 34, 955 (1948).

${ }^{7}$ W. de Sitter, The Astronomical Aspect of the Theory of Relativity (University of California Publications in Mathematics, 1933), Vol. 2, p. 166. See also reference 11. clusters of nebulae are not real clusters, bound together by the gravitational attraction of their members, but just accidental and temporary irregularities in the homogeneous distribution of the galaxies over space."

It might even be that these distributions, although they are not permanent in the sense that the nebulae escape in a fraction of $10^{9}$ years, are nevertheless permanent in the sense that these nebulae going out of the clusters are replaced by other nebulae coming from the field outside. ${ }^{8}$

I have investigated such stationary distributions of nebulae in a field which is itself in equilibrium, that is, a condensation in an Einstein's universe. The main result of this investigation, a result which is probably independent of some simplifying assumptions which have been introduced to make the problem manageable, is that there is a relation between the size of the cluster and the velocity of the nebulae. For velocities of $c / 500$, the dimensions of the condensation is of five to six million light years, somewhat bigger but not of an essentially different order of magnitude than that of real clusters. The result of this investigation cannot be applied directly to the case of the expansion; also the condensations studied are much weaker with central condensation five or six times the average value outside the clusters. An extension of this work to the non-static case is needed and although work is in progress, results are not yet available.

\section{COSMIC RAYS}

The great velocity of the nebulae inside the cluster of about one thousand kilometers per second, shows that the gaseous clouds from which they have been formed had relative velocities of this order. This is a consequence of the fact that the velocity of a nebula is equal to that of the accidentally larger cloud which has occasioned the condensation and retained other of the same speed.

The velocities of the field nebulae have been reduced to one tenth on account of the increase of the radius of space; but the relation between density and velocity is the same for the exchange of nebulae between clusters and field as it is for the general expansion of space. In both cases it is an application of Liouville's theorem of the conservation of density in phase space. Therefore, if the density of the clusters is the density of equilibrium, their velocities are also the velocities at the time of equilibrium.

It is rather difficult to understand what is the origin of this assembly of gaseous clouds with such great relative velocities.

We do not know their density and their size, but we know that the mean free path was great enough so that collisions were rare at equilibrium but became effective in the collapse towards the center.

8 G. Lemaître, "Modèles mécaniques d'amas de nébuleuses," Bull. de l'Acad. Roy. de Belgique, classe des sciences, 34, 551 (1948). 
When the radius of space was smaller, it seems unavoidable that a time could be reached where the clouds should have been in contact one with the other and have formed a unique mass of gas. But such an hypothesis is untenable because there would be no possible way for this mass of gas to split into clouds and to acquire relative great velocities. At that time these velocities should have been even greater because they have been reduced since in the ratio of the expansion.

The only way out is that these gaseous clouds did not exist when the radius of space was much smaller than the equilibrium radius. We must look for some process from which they could have originated.

We are therefore led back to discuss an hypothesis that I proposed eighteen years ago,${ }^{9}$ that cosmic rays existed before the gaseous matter from which the stars are formed so that the gaseous clouds result from the condensation and the absorption of cosmic rays.

I wrote at that time "a possible test of the theory is that, if I am right, cosmic rays cannot be formed uniquely of photons, but must contain, like the radioactive rays, fast beta-rays and alpha-particles, and even new rays of greater masses and charges."

In fact, all kind of matter must be present in the cosmic rays and matter is nothing else than condensed cosmic rays.

These super alpha-rays, formed of every kind of nuclei and not simply of helium as ordinary alpha-rays, have recently been found in the cosmic rays and there is no reason to doubt that all atomic elements exist in the rays. ${ }^{10}$

We can therefore understand that when the radius of space was smaller than $R_{E}$, the gaseous clouds were much more tenuous and would not collide too often. They would continuously absorb cosmic rays and increase their size by capture of these cosmic rays.

At an earlier stage there would be nothing else but cosmic rays. If we can understand the formation of a small mass of gas, just sufficient to produce an absorption equivalent to that of a few grams of air per $\mathrm{cm}^{2}$,

${ }^{9}$ G. Lemaître, Nature 128, 704 (1931).

${ }^{10}$ Freier, Lofgren, Ney, Oppenheimer, Bradt, and Peters, Phys. Rev. 74, 213 (1948). this would be sufficient to produce the clouds of gases.

Of course, clouds evolved in that way would have enormous relative velocities, the velocities of the cosmic rays which happened to encounter one another so gently that they could undergo elastic collisions and make the first steps toward the establishment of the stastical equilibrium characteristic of the gaseous state.

It is well known that energy considerations are in favor of this interpretation. The energy of the rays is estimated to about $0.003 \mathrm{erg} / \mathrm{cm}^{2}$. This is equivalent to $10^{-34} \mathrm{~g} / \mathrm{cm}^{3}$.

For these rays the proper mass is not very different from the energy mass. It would seem that only one ten thousandth of the original cosmic rays escaped absorption and that the rest is condensed or trapped by the clouds and finally transformed into stars and nebulae.

If we consider not the proper energy or mass, but the total energy, then we must take care that the energy of the cosmic rays now observed has been reduced in the ratio of the expansion so that, when the clouds were formed, the energy mass of the rays was much greater than their proper mass.

If for these extreme energies some materialization process may occur which turned kinetic energy into hydrogen or even helium, then the proportion of the rays which is needed for the formation of the matter of the stars is much smaller because atoms of weight higher than helium only should have to be accounted for. Their mass would not be much greater than that of the rays.

In other words, hydrogen and helium found in the stars in so large an amount would have been produced by materialization of kinetic energy of the rays which have been trapped in the formation of gaseous matter. ${ }^{11}$

Of course, the test of this theory would be that if protons are found in the primary cosmic rays, which arrive at the top of the atmosphere, the proportion of these protons as regards the super alpha-rays, formed of heavier matter, is much smaller than the proportion of hydrogen to heavy matter in the stars.

\footnotetext{
${ }^{11}$ G. Lemaître, "Rayons cosmiques et cosmologie," Gazette astronomique, pp. 1-14 (1949); also E. Nauwelaerts Louvain (1949).
} 